\title{
Effect of Acute Alcoholic Liver Injury on Electrical Impedance in Rat Liver
}

\author{
Yitong Song, Yinglu Yao, Huiyingxin Guo, Wenjie Hu, Yinfang Xu, Zhanxiu Cai* \\ Weifang Medical University, Weifang, China \\ *Corresponding author. Email: cai.zhan.xiu@163.com
}

\begin{abstract}
Bio-impedance technology is a new detection technology used to extract medical information concerning the physiological and pathological conditions of tissues based on the electrical properties and changes of cells in biological tissues. This article discusses the development history, characteristics and application of impedance technology, and the methods using Agilent impedance analyzer E4990A in laboratory to measure impedance with four-electrode method, so as to find the changes of electrical impedance in rat liver under acute alcoholic liver injury conditions through modeling and spectrum characteristic curve of impedance. According to previous researches, it has proved that electrical impedance technology can be adopted for the detection of liver and other biological tissues, and the result of research also shows that electrical impedance technology is expected to be used for rapid detection of acute alcoholic liver injury.
\end{abstract}

Keywords: Electrical impedance, Bio-impedance technology, Acute alcoholic liver injury.

\section{INTRODUCTION}

Liver is an important organ with metabolic function in animals, plays a major role in many physiological and biochemical reactions in animals, however, a series of liver injuries may be induced by external trauma or improper living habits. Liver injury generally refers to the damage to liver cells, which alters or impairs the liver structure or function. Liver injury generally includes the trauma-induced liver injury and various liver diseases related to internal medicine system. The causes of liver injury related to internal medicine system shall include viral hepatitis, alcoholic hepatitis and drug-induced hepatitis, among which, alcoholic hepatitis is a very common cause of liver injury.

Drinking culture is omnipresent in China and people are prone to a series of diseases caused by heavy alcohol use during daily life, and alcoholic liver disease is one of the representative diseases. Alcoholic liver disease, also known as alcoholic hepatitis, refers to acute inflammation of the liver caused by excessive alcohol drinking in a short period of time. Patients with the disease will have signs and symptoms such as bleeding and ascites, and the indicators of liver function in biochemical examination will go up. In severe cases, patients will die of acute alcoholism. In recent years, among the inpatients who suffer from liver diseases in the same period, the percentage of alcoholic liver disease cases has been rising. Excessive drinking is associated with significant risks to people's health, so alcoholic liver injury becomes a great concern.

Electrical impedance measurement technology is adopted to extract pathological and physiological conditions of tissues and other biological information based on the electrical characteristics and changes of biological cells. It attracts extensive attention of the scholars at home and abroad due to its advantages of non-invasive, low-cost and easy operation; Many scholars have also made great breakthroughs in terms of detection of cervical cancer, prevention of cancer and disease diagnosis etc. [5]. As alcoholic liver injury may damage liver cells, thus causing significant changes in liver electrical impedance, in this experiment, we study the specific effect on electrical impedance in rat liver under acute alcoholic liver injury starting from alcoholic liver injury.

\section{DEVELOPMENT AND MEASUREMENT OF BIO-IMPEDANCE TECHNOLOGY}

\subsection{Development of bio-impedance technology}

Bio-impedance technology is a detection method used to extract biological information related to 
pathological and physiological conditions based on the difference of conductivity between blood cells and electrolyte solution in biological tissues. Bioelectrical impedance technology was first introduced at the end of the 18th century, and has developed for hundreds of years since then. As the impedance technology gradually develops, Thomastt was the first person applying bioelectrical impedance analysis method to determine the total body water in 1962 [1]; Later, based on further research, a research team in a British university created the first system for measurement of the electrical impedance data of human body in 1987; All these have laid a firm foundation for the improvement and development of bio-impedance technology. In 1992, Matthie et al. proposed a more accurate measurement method, so as to collect more abundant biological information on impedance, so it is also called BIS (Biological Impedance Spectroscopy) [2]. BIS analysis method analyzes these complex impedance characteristics, thus obtaining the biological tissue components based on the impedance electrical characteristics of biological tissues. Similarly, by using BIA method (Biological Immunity Analysis), the body is divided into conductive body fluids, fats, etc., and it can measure the extremely small current emitted by electrode plate passing through body so as to obtain the biological resistance measured. A few years later, Patel [3] and Kushner [4] et.al. adopted the BIS method to measure the content of extracellular fluid, which could get more accurate value compared with BIA method.

China's impedance measurement technology started relatively late compared with foreign countries, but it has made many achievements during its rapid development. In 1993, the Air Force Medical University(AFMU) started the research on bioelectrical impedance tomography, and students Feng $\mathrm{Fu}$ and Xiuzhen Dong, majoring in biomedical engineering of the university, established a biological impedance measurement system based on four-electrode method; In 2007, Tao Han et al. used the human body analyzer to generate the multi-frequency excitation signals through software programming control [6], thus facilitating the establishment of spectrum measurement system. In 2009, Shiqiang Luo et al. developed a kind of electrical impedance spectroscopy measurement system so as to realize measurement of bio-impedance values within the range of $1 \mathrm{~Hz}-10 \mathrm{MHz}$ [7]. Later, the spectrum measurement system gradually enables multi-point measurement. In 2012, Xiue Gao et.al. designed a set of system suitable for multi-point frequency bioelectrical impedance measurement, which adopts a segmented model of the human body and uses DDS chip AD9850 to generate sinusoidal AC signals of different frequencies [8]. In 2020, Haiming Lv designed a kind of bio-impedance multi-frequency detection system used to build DDS inside the FPGA to generate multi-frequency excitation signals suitable for bio-impedance measurement of human body and realize the measurement of multi-frequency electrical impedance of human body [9].

\subsection{Electrical impedance measurement method}

In the laboratory, the electrical impedance of biological tissues is usually measured based on conductivity measurement. It mainly includes two measurement methods, two-electrode method and four-electrode method. According to two-electrode method, only two electrodes are used to measure impedance, and the injection of current signal and the detection of voltage signal are realized using the same pair of electrodes, which means, the needle-shaped electrode at both ends can be deemed as exciting electrode and measuring electrode. Therefore, it can easily cause polarization of electrodes after electric field is generated by adding exciting current; In addition, in terms of the external measurement circuit, the polarization-induced impedance will be affected by the contact impedance, so its measured value does not merely refer to the electrical impedance of the measured tissue, which may produce large errors, and its measured result is usually greater than the true value, indicating the poor accuracy [10].

Considering the imperfection of the two-electrode method, the scholars have developed the four-electrode method. The four-electrode method is the method that is improved based on the traditional two-electrode method. The four-electrode method includes a pair of current exciting electrodes placed outside the biological tissue and a pair of voltage measuring electrodes inserted inside the biological tissue. According to the four-electrode method, independent current electrode and voltage electrode can help reduce the measurement error caused by polarization in the process of solution measurement, as well as reduce the impact of contact resistance. Considering the low electric current passing through the sensing line, the measured resistance value is more close to the true value [11]

\section{CHARACTERISTICS AND APPLICATION OF BIO-IMPEDANCE TECHNOLOGY}

\subsection{Advantages of bio-impedance technology compared with other detection methods}

Currently, the following molecular biological methods such as nuclear magnetic resonance scanning, computed tomography, pathophysiology etc. can be used to examine the physiological and pathological conditions of biological tissues. Although nuclear magnetic resonance technology can show abundant diagnostic information, realize 3D imaging [12] and multi-parameter imaging, it is difficult to realize large-scale promotion due to its high detection cost. 
Although computed tomography has features of high spatial resolution, it can only identify tissues that are different in density. Although it can diagnose benign or malignant results using conventional pathophysiology method, it belongs to static observation and fails to realize real-time monitoring. Therefore, it is particularly important to find a measurement method that can obtain more accurate results with low cost and can identify the tissues indistinguishably.

Bio-impedance detection technology is deemed as a new measurement technology that develops rapidly in recent years, and has attracted many scholars due to its advantages. The bio-impedance technology has no damage or side effects to human body, so this detection method can reflect human health status and pathological information through the electrical impedance information of biological tissues obtained. In addition, it has many other advantages, such as non-invasiveness, no radiation, simple implementation, strong function, unlimited application scenarios, etc. and is applicable to the diagnosis of craniocerebral injury, breast cancer, cervical cancer etc.

\subsection{Application of bio-impedance technology in medical field}

Due to the unique advantages in terms of cost and function, the bio-impedance technology plays an increasingly important role in the medical field, and can be used for the prevention and detection of diseases of various organs and tissues of human body. For example, in the brain monitoring field, the Holder experiment of UK UCL University is conducted to have made great efforts for impedance detection of epilepsy; In China, the Air Force Medical University (AFMU) has also developed a bedside monitoring system applicable to brain image monitoring [13]. Similarly, bio-impedance technology also benefits many women. In foreign countries, T-SCAN technology developed by Israel TransScan Company has developed the breast tumor detection system using the impedance distribution measurement method, which has been applied in the United States [13]. In China, the breast cancer diagnosis technology etc. is also undergoing rapid development. The School of Electrical and Information Engineering, Tianjin University has achieved good achievements in identifying breast cancer focus through bio-impedance technology. The breast cancer focus identification system developed by the School uses the self-made electrodes, and according to the data obtained based on measurement on measurement platform built by impedance analyzer, the experimental results are more accurate than that obtained by BP system developed by Israel Dune [13].

Professor Xiuzhen Dong's team from the Air Force Medical University (AFMU) has also achieved good results. The electrical EISI (impedance scanning imaging) breast detector developed by the team is a kind of technology used to diagnose breast tumors in early stage. By using this technology, we can obtain information and form image based on tissue level, so it is more sensitive for the diagnosis of early breast cancer compared with other diagnostic techniques. This technology can remedy the defects of molybdenum target x-ray mammography and is more suitable for universal screening. This technology is registered as category of national medical instrument, has been tested across the country and achieved remarkable and effective results [14].

\subsection{Existing problems}

During bio-impedance measurement using the bio-impedance technology, we can find many factors affecting the results of impedance measurement. As different tissues in human body show electrical properties, the directivity of current spatial distribution is not strong, and many unknown factors exist in distribution, so it may cause problems such as slightly poor accuracy and poor positioning [15]; Secondly, the impedance real part and imaginary part of biological tissue contain rich impedance information, however, it is not suitable to attract the information of imaginary part of complex impedance and many physiological and pathological information may be lost due to neglecting the influence of phase [16]. Bio-impedance measurement technology is also found with problems in terms of measurement frequency, excitation mode, exciting signal acquisition, measuring electrode material and shape, etc., so it needs to further study the measurement accuracy and driving mode.

Meanwhile, the bio-impedance technology is widely used in the fields such as the electronics, chemistry etc., so it is urgently required to have more comprehensive understanding of the direct or indirect relationship between biological cells, tissues, organs and electrical impedance [17].

\section{DATA ANALYSIS METHOD}

\subsection{Impedance data analysis}

It analyzes the measurement quantities based on impedance spectrum characteristics. According to the electrical impedance experiment [18], there is a big difference in terms of the dielectric characteristics between the normal tissues and diseased tissues, so we can use fitting coefficient and formula [19] to convert the impedance value measured by the impedance analyzer into the conductivity and dielectric constant for weighted fitting, then analyze the fitted data, analyze the conductivity values of the control group and the experimental group respectively within different frequency ranges, so as to infer whether the tissue is abnormal by comparing the size. 


\subsection{Modeling analysis}

Simulation modeling, as a commonly used data analysis method, can realize the visualization of tissues and organs, and allow people to get the required biological information in am more intuitive manner. For simulation modeling, final model imaging is usually realized using calculation formula and other imaging technologies. The method has wide applications, for example, the biological impedance spectroscopy tomography (BIST) integrating electrical impedance tomography and biological impedance spectroscopy is used in the experiment for the purpose of collecting EIT voltage data through electrodes uniformly distributed in measured tissues and reconstructing the conductivity distribution map. According to the conductivity distribution map, the information such as location and size of the lesion area of tissues and organs can be obtained; through the impedance spectrum analysis, category information of lesion area can be obtained. Finally, by integrating the information obtained from conductivity distribution image and impedance spectrum, the location information of measured tissue can be displayed in the image, so as to complete the detection [20]. As this method can visualize the target area and accurately identify the target types, it can be used for early detection of lung cancer or assist clinicians in accurate detection of early lung cancer and improvement of the cure rate of lung cancer in early stage [21].

\subsection{Fitting error analysis of spectrum characteristic curve}

Cell consists of cell membrane and cell fluid, so bio-impedance form is a complex form $\mathrm{Ze}=\mathrm{Re}+\mathrm{JXe}$, with its real part as resistance and its imaginary part as reactance. Bio-impedance results vary a lot within different frequency excitation range, which means, the real part and imaginary part of bio-impedance will change accordingly; Imaginary part is deemed as ordinate and under different electrode spacing, in order to measure the influence of impedance spectrum characteristic curve, a coordinate axis can be established, with real part as abscissa and imaginary part as ordinate, then the residual error fitted by least square method adopted in mathematics is taken as evaluation standard. The better the fitting degree, the smaller the residual sum of squares [22]. This method can be used to analyze blood or local muscle tissue.

\subsection{Statistical treatment}

SPSS statistical software can be used for data analysis and comparison of differences between the experimental group and the control group, and the data are expressed in the form of average value + standard deviation $(\overline{\mathrm{x}} \pm \mathrm{s})[23]$.

\section{CONCLUSIONS}

The electrical impedance technology has an attractive development prospect. With the function of detecting the electrical properties of biological tissues, it can be applied to the disease diagnosis etc. as well as provide possibilities for clinical applications such as lung and brain monitoring [24]. However, bio-impedance technology is still under improvement and has some shortcomings in imaging system and technology. For example, due to the problem of mathematical and physical algorithm, it is insensitive to the specific detection center area, or has the problem of practical application of electrodes, which may restrict the development of impedance technology [25]. In terms of its development, resolution can be improved by perfecting the imaging model and algorithm of biological electrical impedance tomography (EIT), which, meanwhile, can enlarge its advantages, such as amplifying functions, and develop and master holographic impedance method, thus greatly improving EIT image function. After reaching such development stage, the clinical application effect of EIT functional image will be incomparable, which cannot be achieved by using $\mathrm{CT}$ and ultrasound technology [15]. In brief, the impedance technology has a bright future, and it is believed that people will gradually get huge social benefits and economic benefits brought by the development of impedance technology.

\section{ACKNOWLEDGMENTS}

The research is funded by the topics such as the innovative training program for college students in Weifang Medical University: Electrical impedance research on pulmonary fibrosis caused by nasal dripping of bleomycin (X2021493); Preliminary diagnosis of neck muscle strain based on bioelectrical impedance (X2021495); Preliminary research on intracranial pressure monitoring using bioelectrical impedance technology (X2021496): The 13th Five-Year Plan of Shandong Province: Research on the Training Model of Biomedical Engineering Professionals in Medical Colleges Based on the Intersection of Emerging Engineering Education and New Medical Education (2020QZC020); Research Topics on Party Building of Weifang Medical University: New model of ideological and political construction course integrating "frontier teaching + common restricted technology + analog electronic technology engineering education".

\section{REFERENCES}

[1] A T. Bio-electrical properties of tissues [J]. Lyon Med, 1963, 209: 107-118.

[2] Atthie JR WP, Van Loan MD, Mayclin PL. Development of commercial complex bio impedance spectroscopic system for determining 
intracellular and extracellular water volumes. [J]. 1992.p. 203-5.

[3] Patel RV, Matthie JR, Withers PO et al. Estimation of total body and extracellular water using singleand multiple-frequency bioimpedance[J]. Annals of Pharmacotherapy, 1994, 28 (5): 565.

[4] R G, A SD, F KR et al. Single- and multifrequency models for bioelectrical impedance analysis of body water compartments [J]. JOURNAL OF APPLIED PHYSIOLOGY, 1999, 87 (3): 1087-1096.

[5] Jiali Song, Lin Yang, Junjun Kang, Haoting Li, Xiuzhen Dong, Feng Fu. In Vivo Measurement of Electrical Impedance Changes of Brain Tissue in Rats Caused by Ischemic Brain Injury and Histomorphology Control Study [J]. China Medical Device, 2017, 32 (01): 18-21+25.

[6] Tao Han, Yining Sun, Zhiming Yao. Design of Human Body Composition Analyzer--The Realization of Bioelectrical Impedance Principle [J]. Beijing Biomedical Engineering, 2007, 26 (006): 629-633.

[7] Shiqiang Luo, Hua Tian, Hua Huang. Bioelectrical Impedance Spectrum Measurement System based on Single-chip Microcomputer [J]. Modern Electronic Technology, 2009, 32 (12): $146-148+151$.

[8] Xiu'e Gao, Jia Tang, Bo Chen. Multi-frequency and Multi-band Human Bioelectrical Impedance Measurement System [J]. Measurement and Control Technology. 2012, 31 (04): 122-124+129.

[9] Haiming Lv. Research on Multi-frequency Detection Method for Bio-impedance Synthesis of Intracellular and Extracellular Fluids of Human Body [D]: Shenyang University of Technology, 2020.

[10] Weichen Qian. Electrical Impedance of Biological Tissues- Temperature Characteristics and Measurement [J]. Electronic Production, 2019 (22): 71-72.

[11] Wei Han, Haiming Ding, Lingyu Ma. Discussions on Conductivity Meter Measurement Technique using Four-electrode Method [J]. China Metrology, 2003 (06): 63.

[12] Lei Shi. Research on 3D Modeling Method of Silicone Breast Implant after Breast Surgery [J]. Automation Technology and Application, 2021, 40(11): 173-176.

[13] Shiwei $\mathrm{Xu}$; Xiuzhen Dong; Xuetao Shi, Application of Bioelectrical Impedance
Technology in Clinical Research [C], Report Delivered on the 30th Anniversary Conference of Chinese Society of Biomedical Engineering and 2010 Academic Conference of Chinese Society of Biomedical Engineering, 2010, undefined

[14] Canhua Xu, Xiuzhen Dong. Bioelectrical Impedance Tomography and Advances on Clinical Research [J]. High Voltage Engineering, 2014, 40(12): 3738-3745.

[15] Chaoshi Ren; Yunli Cui; Xuedong Chi; Huiyan Wang; Guojing Lin, Problems, Development and Application Prospect of Medical Electrical Impedance Technology [J], Chinese Journal of Medical Physics, 1997, 59-61, 59-61.

[16] Guizhi Xu, Shuo Yang, Ying Li, et.al. Summary of Electrical Impedance Tomography. Journal of Hebei University of Technology. 2004, 33(2): 35-40.

[17] Zhu Zhou; Xiaoyu Li; Wei Wang; Jun Zhang, Research Progress of Bio-impedance Technology and its Applications [C] - Energy Saving and Environmental Protection Harmonious Development - Collected Papers of 2007 Annual Conference of China Association for Science and Technology (III), 2007, undefined.

[18] Zhiyuan Tang, Research on Electrical Impedance Spectrum Characteristics of Hepatoma Carcinoma Cell. Zhejiang Province, Ningbo University, 2015-04-17.

[19] Zhanxiu Cai, Xuetao Shi, Ting Wang, Zhenyu Ji, Feng Fu, Xiuzhen Dong. Research on Dielectric Properties of Normal Breast Fat and Gland Tissue [J]. Chinese Medical Equipment Journal, 2012, 33(05): 1-3.

[20] Hongrun Yin; Ming Ye; Yang Wu; Kai Liu; Huaping Pan; Jiafeng Yao, Biological Tissue Detection Method Based on Biological Impedance Spectroscopy Tomography, Acta Physica Sinica, $1-14,1-14$.

[21] Hongrun Yin, Ming Ye, Yang Wu, Kai Liu, Huaping Pan, Jiafeng Yao. Biological Tissue Detection Method based on Biological Impedance Spectroscopy Tomography [J/O Acta Physica Sinica: 1-14 [2021-12-03]. http://kns.cnki.net/kcms/detail/11.1958.04.202111 16.2233.060.html.

[22] Qinpeng Fu; Yinbao Zhong; An Zhao; Peng Zhao; Wencai Pan; Jieshi Ma. Research on Noninvasive Electrode Spacing Suitable for Local Electrical Impedance Measurement of Muscle Tissue in 
animals [J]. China Medical Device, 2017, (08): $38-41+54$.

[23] Dan Gao, Yuan Zheng, Ran Jiang, Bo Chen, Shaolin Tian, Rong Wang, Wenliang Lu. Effect of Breast Biopsy Method on Sentinel Lymph Node Biopsy [J]. Journal of Clinical Surgery, 2020, 28 (09): 848-851.

[24] Yongjie Zou, Chao Zhang, Weixiang Chen, Hongfei Ge, Shuixian Zhang, Dan Liu, Minglian He, Binbin Tan, Yujie Chen, Hua Feng, Rong Hu, Tangmin Wen. Monitoring Research on Non-invasive Encephaledema Dynamic Monitor based on Bioelectrical Impedance Technology in Macaque Cerebral Hemorrhage and Hematoma Enlargement Model [J]. Journal of Clinical Neurosurgery, 2020, 17 (06): 675-679.

[25] Xin Liu, Chaoshi Ren. Several Bioelectrical Impedance Tomography Methods and Systems under Development [J]. Chinese Medical Equipment Journal, 2008(10): 33-35+38. 